\title{
Internet en el ámbito del Trabajo Social: formas emergentes de participación e intervención socio-comunitaria
}

\author{
The Internet in the field of Social Work: emerging forms of participation \\ and socio-communitarian intervention
}

\author{
Rubén ARriAzU MuÑoz \\ Universidad de Extremadura \\ rarriazu@unex.es \\ José Luis FERNÁNDEZ-PACHECO SÁEZ \\ jlfpsaez@trs.ucm.es
}

Recibido: 13/02/2012

Revisado: 30/05/2012

Aceptado: 30/07/2012

Disponible on line: 16/04/2013

\begin{abstract}
Resumen
Internet constituye por sí mismo un medio de comunicación que altera modifica y reestructura los procesos de interacción y socialización de un individuo, grupo o sociedad. El progresivo uso de las herramientas comunicativas on-line (foros de discusión, listas de correo, IRC-chats, etc.), ha motivado por su propia funcionalidad y redefinido los patrones comunicativos ortodoxos en el espacio-tiempo. Aplicando este planteamiento al ámbito del Trabajo Social surgen cuestiones importantes tales como: ¿constituyen estas herramientas de comunicación virtuales un instrumento con el que fomentar y dinamizar la participación socio-comunitaria?, ¿podrían incluirse estas herramientas dentro de las propuestas metodológicas de intervención en el ámbito de Trabajo Social?, ¿qué papel jugará internet, y concretamente, las herramientas de comunicación on-line en el ámbito del Trabajo Social de las próximas décadas?

El objetivo del presente artículo es abordar estas preguntas desde un punto de vista global y sistemático, analizando las ventajas y los inconvenientes que plantea actualmente el uso de las tecnologías de la información y comunicación (TICs) en el ámbito de Trabajo Social. Centramos la atención en la implicación, que históricamente ha tenido, y tiene la tecnológica en los procesos de participación ciudadana e intervención social, reflexionando sobre la potencialidad de estas herramientas para la práctica profesional. Se trata, en definitiva, de analizar la viabilidad que poseen los recursos tecnológicos comunicativos en el marco de las competencias profesionales de Trabajo Social.
\end{abstract}

Palabras clave: Trabajo Social on-line, participación comunitaria on-line, herramientas de intervención on-line, voluntariado on-line, comunicación mediada por computadora (CMC).

\begin{abstract}
The Internet, in and of itself, constitutes a media that modifies and restructures the processes of interaction and socialization of an individual, group or society. The increasing use of online communication tools (Discussion Boards, LISTSERV email lists, IRC chats, etc.) has motivated, by their functionality, the redefinition of orthodox models of communication in space and time. By applying this concept to the field of Social Work, important questions arise, such as, Do these virtual tools of communication constitute an instrument with which to foment and invigorate socio-communitarian participation?, Could these tools be included within methodological proposals for intervention in the field of Social Work?, What role will the Internet, and more specifically, on-line tools of communication, play in Social Work in the coming decades?

The objective of this paper is to address these questions globally and systematically, analyzing the advantages and disadvantages of the use of Information and Communication Technologies («ICTs») in the field of Social Work. We focus attention on the implications that technology has, and historically has had, for the processes of public participation and social intervention, reflecting on the potentiality of these tools for professional practice. We seek to definitively analyze the viability of these technological communication resources within the framework of professional competencies of Social Work.

Keywords: on-line social work, on-line communitarian participation, on-line tools of intervention, on-line volunteer service, Computer-Mediated Communication (CMC).
\end{abstract}

Referencia normalizada: Arriazu Muñoz, R., y Fernández-Pacheco Sáez, J. L. (2013): «Internet en el ámbito del Trabajo Social: formas emergentes de participación e intervención socio-comunitaria». Cuadernos de Trabajo Social, 26(1): 149-158.

Sumario: Introducción. 1. Herramientas de comunicación virtual aplicadas al Trabajo Social. 2. La participación y dinamización social a través de Internet. 3. Estrategias de intervención social a través de Internet. 4. Conclusiones. 5. Referencias bibliográficas. 


\section{Introducción}

Desde un planteamiento convencional, la lógica del diseño tecnológico ha estado orientada a la mejora de las condiciones vitales del individuo. En la práctica no siempre ha sido así, el desarrollo tecnológico no ha resultado un proceso homogéneo y accesible, ni para todas las personas, ni en todos los contextos socioculturales. Consecuentemente, la implantación de estos sistemas tecnológicos, y especialmente, los que afectan a las Tecnologías de la Información y Comunicación (TICs) ha provocado realidades duales, donde los individuos que no acceden o poseen este tipo de servicio o herramienta o recurso quedan relegados a planos subyacentes de aislamiento en la Sociedad Global del Conocimiento. Sobre esta cuestión, Alvin Toffler pone de manifiesto la idea de que los analfabetos del siglo XXI no serán los que no puedan leer y escribir, sino quienes no puedan aprender, desaprender y volver a aprender (Toffler, 1996). Sin embargo, llegados a este punto, conviene, no solamente quedarse en el escenario catastrofista de que describe este autor, sino incidir en el condicionante previo de aquél; es decir: ¿qué lugar ocupan las personas en el mundo si no tienen acceso a las TICs?, y en el caso de que tuviéramos acceso, ¿cómo resulta el proceso de aprendizaje y aplicación de las TICs al ámbito personal, social y profesional?

Tomando en consideración esta premisa, que ha dado lugar a la atribución de sobrenombres como «analfabetos digitales para aquellas personas no vinculadas al uso de las TICs» (Ortoll, 2007, p. 85), la propuesta analítica de este texto pretende examinar las potencialidades del uso de estas tecnologías para las profesiones relacionadas con la intervención social, como es el caso del Trabajo Social, prestando especial atención a dos elementos interdependientes: por un lado, los procesos de participación y dinamización social, y por el otro, las metodologías de intervención social aplicadas al estudio de caso. Esta primera aproximación pretende ser una reflexión que invite a seguir investigando, analizando, y en definitiva, reformulando la lógica de la intervención social a partir de los recursos tecnológicos existentes. La generación de nuevas alternativas de intervención deberá llevar implícito, no solamente un conjunto de propuestas para la práctica, sino también una reflexión teórica que asuma o ponga en cuestión los planteamientos epistemológicos tradicionales ligados a un modo de proceder, en este caso, las metodologías de intervención social.

Desde un punto de vista retrospectivo, los avances tecnológicos en la comunicación han estado más o menos presentes en determinados modelos de ayuda mutua e intervención social. Históricamente, el teléfono constituyó uno de los primeros ejemplos tecnológicos básicos que ha facilitado y agilizado el desarrollo de las prácticas profesionales. Si pensamos cómo sería hoy el despacho de un trabajador o una trabajadora social sin este aparato, cómo habría cambiado su práctica profesional cotidiana, seguramente tomaremos conciencia de su centralidad en la práctica profesional. Superando el nivel instrumental, y profundizando en los procesos de intervención social y ayuda, el teléfono ha servido y sirve como herramienta comunicativa en la intervención psicosocial. Asociaciones como «El Teléfono de la Esperanza», «SOS ayuda al maltrato», o el «Servicio de Teleasistencia» ${ }^{1}$, utilizan actualmente este recurso como elemento central de acceso y cobertura de los servicios que prestan.

Aplicando esta misma lógica cabe preguntarse: ¿puede Internet, entendido como un medio de comunicación, contribuir de forma activa a los procesos de participación y dinamización sociocomunitaria? Desde el punto de vista del Trabajo Social, ¿qué ventajas e inconvenientes plantea la intervención social a través de Internet? Para dar respuesta a estas cuestiones es necesario profundizar en los beneficios y riesgos que ofrece el espacio virtual como medio comunicativo examinando las singularidades que presenta la práctica profesional cotidiana.

${ }^{1}$ Para ampliar información sobre estos servicios se remite al lector a las páginas web de estas asociaciones donde se explica detalladamente el funcionamiento de este tipo de asistencia:

1. Teléfono de la esperanza [Documento WWW] URL http://www.telefonodelaesperanza.org/llamanos

2. SOS ayuda al maltrato [Documento WWW] URL http://seguridad.galeon.com/enlaces612482.html

3. Servicio de Teleasistencia [Documento WWW] URL http://www.teleasistencia.com/home/ 


\section{Herramientas de comunicación virtual aplicadas al Trabajo Social}

Los procesos de participación social a través de Internet están supeditados, en su funcionamiento, a un conjunto de medios o herramientas comunicativas on-line. Estas herramientas han modificado los canales comunicativos preexistentes tanto para el ciudadano común como para los profesionales de una disciplina científica. Prestando atención a este último grupo, es importante examinar la funcionalidad que tienen o pueden tener determinadas herramientas comunicativas on-line para el Trabajo Social. Para ello, atenderemos a dos planos de análisis complementarios: por un lado, a la propia utilidad que reportan estas herramientas para el profesional del Trabajo Social, es decir, cómo los trabajadores sociales pueden hacer uso de este tipo de herramientas para mantenerse vinculados a cuestiones profesionales; y por el otro, desde un plano más orientado al ejercicio de la profesión, se vinculará la utilización de estas herramientas comunicativas a las metodologías de participación y dinamización de grupos sociales aplicadas desde el Trabajo Social.

Una de las taxonomías más consensuadas sobre instrumentos comunicativos en Internet tiene como punto de partida el grado de sincronía que se genera en el acto comunicativo, diferenciando entre herramientas de comunicación sincrónicas y asincrónicas (Yus, 2001). En el caso del primer grupo, la sincronía viene determinada por la confluencia en el espacio y tiempo de los interlocutores, independientemente de su contexto geográfico. Ejemplos de este primer grupo podrían ser los sistemas basados en el protocolo Internet Relay Chat (IRC), comúnmente conocido como chat, o las herramientas integrales de voz y texto basadas en el sistema (Voice IP), utilizadas para realizar videoconferencias. Las herramientas de comunicación asincrónicas en cambio, son aquellas en las que los interlocutores no comparten ni espacio ni tiempo, es decir, son herramientas que no requieren un elevado grado de instantanei- dad entre las partes ya que se da por hecho la espera en el tiempo de la respuesta. Ejemplos de este tipo de herramientas podrían ser las listas de correo, los foros de discusión y en determinados casos ${ }^{2}$, los sistemas microblogging como pueden ser Facebook, Academia, Twitter, Tuenti, Eskup, LinkedIn, Google+, etc.

Tomando como referencia esta clasificación, que determina los procesos comunicativos según su marco temporal de respuesta, es el turno ahora de describir y analizar la adaptabilidad que presentan este tipo de herramientas en el ámbito del Trabajo Social. El abordaje de todas las herramientas on-line existentes en la actualidad resulta una tarea que trasgrede los límites del texto, por este motivo, hemos seleccionado las que a nuestro juicio, consideramos se ajustan mejor a los procesos de dinamización y participación social.

\subsection{Lista de correo/Mailing list}

Las listas de correo, listas de discusión, también llamadas mailing lists, pueden definirse como un elenco donde se encuentran registradas una serie de personas que comparten o tienen una afinidad o un interés común. Su funcionamiento es bastante sencillo: básicamente consta de un programa donde se almacenan las direcciones de correo electrónico de todas las personas que forman parte de la lista, o mejor dicho, que están suscritas a la misma. Cada vez que una persona envía un mensaje a la lista, este programa se encarga de enviar una copia al resto de personas que están suscritas a ella (Barrueco et al, 1996, p. 23). El envío de este mensaje puede quedarse en un plano divulgativo, es decir, de transferencia de información o, por el contrario, en un plano de debate, donde los miembros del grupo responden a la lista e intercambian diferentes puntos de vista sobre dicha temática. Dentro del ámbito del Trabajo Social, existen un número considerable de listas de correo. Por citar algunas de las más representativas en el caso español, cabría hablar de portales como RedIRIS o ELISTAS ${ }^{3}$, que

\footnotetext{
2 Conviene matizar que determinados sistemas de microbbloging que pueden ser considerados como herramientas de comunicación asincrónica han incorporado en sus portales herramientas de comunicación sincrónica como en este caso los chats.

3 Los enlaces para participar en estas listas de correos se encuentran disponibles en las siguientes direcciones:

[Documento WWW] URL http://www.rediris.es/list/info/tsocial.html y

[Documento WWW] URL http://www.elistas.net/lista/trabajosocial.
} 
ofertan este servicio de manera gratuita desde hace casi una década. En un plano internacional podrían destacarse las listas de correo de la National Association of Social Work (NASW), The New Social Worker (ARNOVA) o de CTISOC-WORK-UK donde se aborda y discute un variado número de ámbitos de intervención relativos al Trabajo Social ${ }^{4}$.

\section{2. Foro de discusión}

El foro de discusión o discussion borrad puede definirse como un espacio virtual al cual accede un determinado número de personas en Internet, con el fin de conocer, producir e intercambiar conocimientos, colaborar y aportar puntos de vistas individuales sobre una temática interesada y comúnmente compartida.

En cuanto a su estructura organizativa, el foro de discusión viene determinado por la figura de un moderador o gestor encargado de supervisar y dinamizar tanto el correcto funcionamiento del grupo de usuarios, como la aplicación informática. De igual forma, la dinámica y funcionamiento del foro de discusión parten de un procedimiento muy elemental: cada usuario accede a la aplicación del foro de discusión, alojada en una página web a través de un nombre y una contraseña. Tras el registro y la autorización del moderador o gestor de la página, se adentra en el espacio virtual donde confluyen un conjunto de temáticas tratadas - comúnmente denominadas hilos de discusión o threads - que a su vez albergan el conglomerado de mensajes - o post - de los distintos usuarios (Arriazu, 2007, p. 6).

En la actualidad existe un amplio número de foros de discusión que abordan diferentes temáticas relacionadas con la acción social y la intervención socio-comunitaria. Uno de los foros de discusión que reúne a un mayor número de profesionales del Trabajo Social anglosajones está alojado en la página web denominada
The new social worker on-line. En el caso español, encontramos varias alternativas menos centralizadas en portales como «trabajosocialya», «trabajo-social.mforos» o, ya en ámbitos más específicos, como por ejemplo «discapnet» ${ }^{5}$. Todos ellos, siguiendo la misma lógica de compartir e intercambiar información y puntos de vistas sobre un problema o temática determinada.

\subsection{Sistema microbbloging}

El sistema microvbloging genera un tipo de comunicación muy concreto, donde el contenido de la información que se desea compartir con el resto de personas se encuentra constreñida, y en algunos casos limitada, por un determinado número de caracteres textuales. Desde un punto de vista más teórico, esta herramienta guarda cierta correspondencia con el planteamiento de modernidad líquida de Bauman (2005), donde lo significativo de la interacción radica en la inmediatez y en lo efímero del momento; es decir, es una herramienta en la que priman los aspectos nucleares de la comunicación en detrimento de las partes explicativas o periféricas. Popularmente, estos sistemas se encuentran incardinados dentro de los servicios que ofrecen las plataformas web 2.0 (O'Reilly, 2004), y especialmente, las populares «redes sociales» como Facebook, Twitter, etc.

$\mathrm{Su}$ funcionamiento, al igual que el foro de discusión, parte de un registro en una página web donde se da de alta un nuevo perfil de usuario. Éste, a su vez, se encuentra vinculado a los perfiles de otros usuarios, bien a través de un sistema de seguimiento (Twitter), bien por un sistema de vinculación a un grupo (Facebook), o simplemente, por algún tipo de relación preliminar en el contexto off/on-line. Lo llamativo de estos sistemas, al igual que el resto de herramientas de comunicación asincrónicas explicadas hasta el momento, es que la informa-

\footnotetext{
4 Para ampliar información sobre el tema se remite al lector a los siguientes enlaces:

[Documento WWW] URL http://www.naswdc.org/pressroom/media/mediaListserve.asp y

[Documento WWW] URL http://isdpp.bnu.edu.cn/isdpp_e_lib/int/contacts/discus_grp_soc_work.htm.

5 Para profundizar sobre esta temática se remite al lector a los enlaces de las páginas web mencionadas.

«The new social worker on-line» [Documento WWW] URL http://www.socialworker.com/home/component/option,com_wrapper/Itemid,106/

«Trabajosocialya» [Documento WWW] URL http://trabajosocialya.mforos.com/

«Trabajo-social.mforos» [Documento WWW] URL http://trabajosocialya.mforos.com/forums/

«Dicapnet» [Documento WWW] URL http://foros.discapnet.es/Default.aspx
} 
ción que da a conocer cada uno de los usuarios se hace visible de manera directa para el resto de personas vinculadas, agregadas, o seguidoras, generándose así diferentes tipos de interacciones. En el ámbito del Trabajo Social, existe un significativo número de grupos afines a la profesión visibles en todas las redes sociales de las que venimos citando hasta el momento.

\subsection{Chat $\mathrm{y}$ sistemas Voice IP}

El protocolo de comunicación Internet Relay Chat (IRC) es la base funcional del chat, una de las herramientas de comunicación sincrónica que goza, desde sus inicios, de un alto grado de popularidad entre los usuarios de Internet. Esta herramienta está basada en la comunicación en tiempo real a través del texto, donde dos o más personas pueden intercomunicarse a través de un canal común alojado en una página web (De Miguel, 2006). Originariamente, esta herramienta se concibió para comunicarse exclusivamente a través del texto, sin embargo, lo cierto es que durante la última década este planteamiento inicial ha ido incorporando mejoras que incluyen sistemas de videoconferencia donde se integran la imagen, la voz y el texto. Estas variantes del chat se basan en el sistema Voice IP y suponen un salto cualitativo en el desarrollo de las herramientas de comunicación en tiempo real. Entre las aplicaciones comerciales más populares que utilizan este sistema cabría destacar a Skype, Google Talk, Yahoo o Messenger y entre las diseñadas con software libre cabría hablar de Yugma, WizIQ, OoVoo, Vyew o DimDim.

A pesar de las singularidades que presentan tanto el chat como los sistemas Voice $I P^{6}$, lo cierto es que este tipo de herramientas suponen una alternativa viable a la comunicación para el ámbito profesional del Trabajo Social, en la medida que permiten articular canales comunicativos más extensos que transgreden los límites geográficos actuales. Gracias a estas herra- mientas de comunicación en tiempo real, hoy en día es posible mantener una reunión de trabajo entre expertos de diferentes puntos del mundo, compartiendo información instantánea $\mathrm{y}$ abordando de manera conjunta situaciones y problemáticas locales en un entorno virtual que resulta, a su vez, global.

\section{La participación y dinamización social a través de Internet}

Una vez definidas, las que a nuestro juicio, constituyen las principales herramientas de comunicación on-line, es turno ahora de profundizar en la utilidad, funcionalidad y aplicabilidad de estas herramientas dentro del ámbito del Trabajo Social, y especialmente, en lo que concierne a los procesos de participación y dinamización social a través de Internet.

El primer elemento a tener en cuenta para generar los procesos de participación y dinamización social a través de la práctica profesional en la red es, sin lugar a dudas, la infraestructura, es decir, que tanto el profesional como las personas con las que se desea trabajar dispongan de acceso y cobertura a Internet. Como señala Bendit (1999) ${ }^{7}$ o Krauskopf (1998), los beneficios del desarrollo tecnológico no favorecen a todos los estratos sociales por igual, por ello y aunque pueda resultar obvio, lo primero que se debe tener en cuenta, antes de plantear el uso de este tipo de herramientas, es la viabilidad para llevarlo a cabo.

En segundo lugar, es importante tener en cuenta la competencia técnica del profesional, dicho de otro modo, que el trabajador o la trabajadora social, posean los conocimientos básicos-instrumentales para poder diseñar o configurar la herramienta comunicativa. Si hace una década la tarea de crear una lista de correo, un foro de discusión, un chat o una página web suponían un reto inalcanzable para los neófitos de la informática, lo cierto es que el desarrollo de las aplicaciones web 2.0 ha mitigado este efecto convirtiéndolo en una tarea relativamen-

${ }^{6}$ Como se ha comentado, los sistemas Voice IP presentan ciertas diferencias respecto al chat, no sólo porque tienen incorporadas las mejoras en la imagen, el sonido y el texto sino porque la interacción se genera a través de un software propio que permite comunicarse a personas de una lista predeterminada por el usuario. Sin embargo, en el caso del chat la dinámica comunicativa se produce en un canal de una página web donde los usuarios pueden, o no, haber tenido un contacto previo entre ellos.

7 La ponencia presentada durante la Reunión del Grupo de Trabajo «Juventud» CLACSO (Buenos Aires, 14- 17.12. 1999), pone el énfasis en el concepto de «diferenciación social» para explicar las diferencias sociales entre los grupos en la sociedad actual. 
1. Acceso y cobertura del profesional y de las personas implicadas

2. Competencias en el diseño o creación de la herramienta comunicativa

3. Dinamización inicial basada en potenciar las capacidades comunicativas

4. Gestión y soporte técnico de la infraestructura

5. Dinamización de proceso basada en el mantenimiento de interacciones

6. Evaluación del proceso de participación y mantenimiento de interacciones

Cuadro 1. Factores y condicionantes de la participación social on-line.

te sencilla gracias a los gestores de contenidos que ya incluye la herramienta ${ }^{8}$.

Solventados los problemas que pudieran surgir en el acceso y las competencias técnicas, la tercera cuestión a tener en cuenta en los procesos de participación social on-line hace referencia a la presentación de la herramienta comunicativa a los participantes. La dinamización inicial debe explicar detenidamente el objeto social de la herramienta haciendo hincapié en las ventajas que reporta la utilización del medio virtual. Por ejemplo, en el diseño de un foro de discusión o un chat para un grupo de ayuda mutua para personas cuidadoras, sería interesante señalar que se trata de un espacio virtual donde poder compartir en cualquier momento y lugar información, problema o simplemente inquietudes, con personas que se encuentran en una situación de similares características.

Igualmente significativo resulta la gestión y el soporte técnico a la hora de gestionar la participación on-line. Es evidente que no todas las personas poseen el mismo grado de capacitación tecnológica. Prensky atribuye un mayor grado de competencia tecnológica a las personas que han nacido durante el período de desarrollo tecnológico - nativos digitales - y no tanto a los que lo hicieron con anterioridad - inmigrantes digitales - (Prensky, 2001). Otra clasificación más actualizada que supera la propuesta biologicista de Prensky es la del británico David White (2011) a través de un modelo donde lo importante no es la edad, sino el grado en el que la persona se halla involucrada en las aplicaciones tecnológicas. Para ello, diferencia entre dos figuras, el visitante que englobaría a las personas que utilizan la tecnología de manera esporádica y con un bajo grado de implicación, y el residente que sería todo lo contrario, es decir, las personas que, independientemente de su edad, tienen un alto grado de implicación con el uso y manejo de las tecnologías de la información y comunicación.

Independientemente de estas clasificaciones, lo que resulta importante, desde el punto de vista de la participación social on-line, es que el profesional atienda las posibles incidencias o problemas de las personas implicadas en el grupo. Debe existir un respaldo que contribuya a consolidar la herramienta como un medio de comunicación válido y fiable para las personas involucradas, y es ahí, donde la figura del profesional cobra una tarea importante en la resolución de problemas instrumentales.

Superado el nivel técnico, otra de las funciones a tener en cuenta por el profesional en los procesos de participación social on-line está relacionada con la dinámica que se genera entre los miembros del grupo. Dependiendo del objetivo que nos planteemos con las personas implicadas, el profesional podrá desarrollar mo-

\footnotetext{
${ }^{8}$ Para ampliar información sobre la creación o configuración de herramientas comunicativas consúltese los siguientes enlaces

Listas de correo: [Documento WWW] URL http://www.rediris.es/list/sol/

Foros de Discusión: [Documento WWW] URL http://www.foroactivo.com.es/ o http://www.my-forum.org/

Sistemas Microblogging: [Documento WWW] URL http://es-es.facebook.com/pages/C\%C3\%B3mocrear-un-grupo-en-Facebook/137988639556674

Chats: [Documento WWW] URL http://es.tinychat.com/
} 
delos o estilos de liderazgo más o menos participativos otorgando mayor o menor grado de independencia. Cualesquiera que fuera el modelo a desarrollar, lo necesario en este caso es que el profesional se ocupe de mantener activos los procesos de interacción y comunicación entre las personas, bien a través de preguntas generales, informando al grupo de noticias afines o mediante cualquier otra técnica que invite a los participantes al uso de la herramienta virtual.

El último de los factores a tener en cuenta en el proceso de participación a través de Internet hace referencia a la evaluación del proceso realizado; es decir, el profesional debería analizar los beneficios e inconvenientes que ha planteado en el grupo la utilización de este tipo de herramientas on-line. Sobre esta cuestión, es interesante profundizar en el grado de relación e interacción que mantienen los miembros del grupo tras haber dado por concluido el uso de la herramienta ya que servirá como indicador para conocer el funcionamiento y el grado de idoneidad del instrumento en los propios participantes.

Esta propuesta de dinamización y participación social parte de un punto de vista centrado en la intervención social del profesional en un ámbito determinado; sin embargo, es importante resaltar que las herramientas de comunicación virtuales, sincrónicas y asincrónicas, son extensibles a otros ámbitos de la acción social. Uno de los ejemplos más significativos que progresivamente va cobrando un impacto social relevante es el voluntariado virtual, ciber-voluntarios u online volunteering, entendido como un tipo de voluntariado que contempla acciones de este tipo realizadas a través de Internet. Estos programas son impulsados principalmente por Organizaciones no gubernamentales, aunque cada vez más organismos internaciones, como Naciones Unidas ${ }^{9}$, desarrollan este tipo de programas como parte complementaria de su política social estratégica.

\section{Estrategias de intervención social a tra- vés de Internet}

Imaginemos que entra al despacho de un trabajador o de una trabajadora social una persona inmigrante recién llegada a España, sin cono- cimiento de nuestro idioma y, consecuentemente, sin capacidad para comunicarse con nosotros: ¿qué se puede hacer? ¿Cómo intervenir ante una situación así?, ¿pueden las tecnologías de la información y comunicación (TICs) ayudarnos en este caso? Cuando tuvimos que enfrentarnos a esta situación real, pensamos en Internet como posible alternativa, accediendo a un traductor on-line. A través del intercambio de frases breves, logramos establecer una comunicación lo suficientemente fluida que nos permitió entender y atender a sus necesidades. Este lacónico, pero representativo, ejemplo debe servir para preguntarnos: ¿pueden las TICs, y especialmente Internet, contribuir de forma activa en los procesos de ayuda, y concretamente, en la intervención social? ¿Qué ventajas e inconvenientes plantea la utilización de estos recursos comunicativos en la intervención a través de Internet?, y su utilización dentro del ámbito de trabajo ¿reformula o mantiene la actual metodología de intervención social ortodoxa?

\section{1. ¿Cuándo llevar a cabo una interven- ción social a través de internet?}

Lo primero que conviene dejar claro es que internet, y con ello la intervención social on-line, constituye una alternativa al modelo de intervención social convencional, sin embargo, en ningún caso la pretensión de este texto es plantear este procedimiento como una vía excluyente para desestimar la metodología de intervención presencial. Internet, entendido como medio de comunicación, habilita un espacio de ayuda de carácter individual o grupal que sirve para extender y ampliar las competencias profesionales ortodoxas del Trabajo Social.

Si bien no existe una fundamentación explícita o protocolo de actuación para desarrollar este tipo de tratamientos, lo cierto es que existen casos y circunstancias en que la aplicación de las intervenciones sociales on-line permite superar y sobreponer determinados hándicaps que afectan al desarrollo normal de una intervención social cara a cara. Partiendo de las limitaciones que supone abarcar la complejidad social en su conjunto y atendiendo a los condicionantes sociales y psicológicos que pueden

${ }^{9}$ Para profundizar en el programa de voluntariado virtual de Naciones Unidas consúltese el siguiente enlace. [Documento WWW] URL http://www.onlinevolunteering.org/es/index.html 
darse en una intervención social convencional, las intervenciones sociales on-line quedarían justificadas en los siguientes supuestos:

1. Intervención social on-line cuando existan inconvenientes en el proceso comunicativo: este tipo de intervenciones englobarían a aquellas situaciones donde el profesional o la persona demandante presenten algún tipo de obstáculo que dificulte el proceso comunicativo. Por ejemplo, personas con discapacidad auditiva o lingüísticas que encuentren en la escritura a través de Internet el modo más eficaz de transmitir sus necesidades o desarrollar su trabajo con el profesional o la profesional de Trabajo Social.

2. Tratamiento terapéutico de pacientes con problemas de movilidad o determinados trastornos: la intervención social en este segundo grupo se realizaría con personas que presenten problemas de movilidad o posean algún tipo de trastorno o fobia que les impida desplazarse con normalidad a la institución competente.

3. Tratamiento terapéutico con problemática geo-temporal: la intervención on-line se justifica en este caso cuando el profesional o el usuario tienen una problemática determinada que les impide realizar reuniones presenciales en un «espacio-tiempo». Por ejemplo, una mujer que trabaje y esté al cuidado de sus hijos podría tener problemas para mantener sesiones periódicas con el profesional.

4. Intervención social en situaciones de riesgo: la intervención en este último grupo tendría lugar cuando las características de la persona demandante supongan un riesgo para afrontar un tratamiento individualizado presencial. Ejemplos de este tipo de tratamiento podría darse en centros penitenciarios donde la regulación interna no permite la intervención presencial con reclusos considerados altamente conflictivos.

Las intervenciones sociales on-line, son actualmente uno de los grades focos emergentes en los procesos de ayuda. En la actualidad cada vez más son las asociaciones no gubernamentales las que aprovechan la potencialidad de Internet, desarrollando acciones de captación de voluntarios virtuales (Peña, 2002, p. 198); sin embargo, la pregunta en este punto es sumamente sencilla: ¿cómo plantear un tratamiento terapéutico utilizando este medio virtual? ¿Cómo intervenir con una persona al margen de la concepción ortodoxa del espaciotiempo? ¿Resulta efectiva una intervención a través de Internet donde el plano virtual se sobrepone al clásico tratamiento cara a cara?

Abordar estas preguntas implica un posicionamiento sobre la conveniencia, o no, de este tipo de herramientas en la intervención social. En cualquiera de los casos, y como se ha señalado anteriormente, conviene dejar claro que Internet, entendido como medio de transferencia, constituye un espacio comunicativo que articula y posibilita diferentes tipos de interacción y comunicación entre el profesional y el usuario. En ausencia de un protocolo sistemático para la intervención social on-line, hemos considerado interesante mencionar las recomendaciones y principios éticos aprobados por la International Society for Mental Health Online (ISMHO) ${ }^{10}$ donde el profesional que presta sus servicios a través de Internet debe tener en cuenta tres elementos básicos que hacen referencia a:

1. El usuario debe ser informado de los servicios que se le van a prestar a través de este medio dejando constancia de los beneficios y riesgos que conlleva este tipo de intervención.

2. El planteamiento de la intervención social debe seguir el mismo procedimiento de actuación que si fuera de modo presencial en términos de competencias, requerimientos para la práctica, evaluación del problema, confidencialidad y evaluación del proceso.

3. El usuario debe conocer los canales de comunicación en situaciones de urgencia. Al igual que el punto uno, este aspecto debe tratarse previamente al comienzo de la intervención.

Estas recomendaciones de partida suponen los primeros intentos por sistematizar el proceso de intervención social on-line, proceso, que a priori, resulta ciertamente complejo si atendemos a la diversidad de casos, perspectivas,

${ }_{10}$ Para ampliar información consúltese el apartado de principios éticos y sugerencias de la página web de la International Society for Mental Health Online.

[Documento WWW] URL https://www.ismho.org/suggestions.asp 
modelos y enfoques desde los que puede plantearse la intervención en el ámbito social. Entendiendo las limitaciones que pueda presentar el contexto virtual en cada caso concreto, señalamos a continuación las principales ventajas e inconvenientes que se derivan de su aplicación.

\section{Ventajas}

— Flexibilidad, accesibilidad y fluidez en la relación entre profesional y usuario.

- Se logra un análisis más exhaustivo del componente verbal, muy valorado en distintos modelos y enfoques de intervención social.

- Facilidad en el registro y sistematización de la información obtenida durante la todo el proceso intervención social.

- Permite articular un modelo de intervención claro y reflexivo donde el profesional y el usuario cuenten con mayor margen temporal para la explicación del problema y su posterior diagnóstico.

- Favorece el anonimato y la confidencialidad del usuario a través de Internet.

- En las últimas fases de la intervención puede utilizarse como vía para deshabituar la relación del usuario con el profesional en la búsqueda de la autonomía integral de la persona.

\section{Inconvenientes}

- Problemas de acceso y cobertura técnica por parte del profesional o persona demandante de ayuda.

- Dificultad para establecer condiciones de empatía con el usuario que permitan profundizar en los problemas latentes.

- Pérdida de la comunicación no verbal que dificulta la comprensión global de los problemas del usuario.

- Necesidad de conocer la normativa legal y jurídica del contexto del usuario si la intervención social se realiza en contextos geográficos diferentes.

- Necesidad de formación especializada de profesionales en el manejo y uso de este tipo de herramientas.

Profundizar en cada uno de estos puntos supone repensar la importancia de aplicar este ti- po de herramientas comunicativas en el ámbito de la intervención social. Si bien los usos y las herramientas comunicativas a través de Internet sufren continuas transformaciones ${ }^{11}$, lo interesante de cara al futuro será adaptar las potencialidades de este tipo de herramientas -en aquellas situaciones que resulte conveniente- a los modelos de intervención social en el ámbito de la acción social.

\section{Conclusiones}

Tomando en consideración todos los argumentos expuestos hasta el momento podemos afirmar que la progresiva utilización de las herramientas comunicativas virtuales en las sociedades modernas condiciona y transgrede la utilización para la que fueron inicialmente diseñadas y ello obliga a repensar su uso desde el punto de vista profesional. Sucintamente, se ha tratado de explicar las aplicaciones prácticas que puede tener este tipo de herramientas comunicativas on-line en los modelos de participación o dinamización social y en las metodologías de intervención social, abriendo la posibilidad de consolidar un foco emergente para el Trabajo Social. Si bien es cierto que la metodología de intervención social tradicional contempla la participación como fundamento básico, lo cierto es que la utilización de estas herramientas transgreden y amplían los espacios geo-temporales abriendo nuevas vías para alcanzar una mayor implicación, continuidad y seguimiento.

Como se ha señalado anteriormente, este tipo de propuestas de interacción virtual se enmarcan dentro de un plano complementario, y en ningún caso excluyente, a las prácticas profesionales presenciales que desde el Trabajo Social tradicional se vienen desarrollando hasta el momento. Entendiendo que este tipo de prácticas se encuentran hoy en día muy condicionadas por la accesibilidad y competencia de las TICs, lo interesante en este punto será seguir reflexionando sobre las potencialidades que ofrece la comunicación mediada por computadora $(\mathrm{CMC})$ en los procesos de participación e intervención social para poder seguir ofreciendo alternativas profesionales en la co-

11 Las herramientas comunicativas a través de Internet buscan cada vez más alcanzar las características de la comunicación presencial. Se persigue, la inmediatez, la sincronía y el tiempo real dentro de un mundo virtual atemporal y deslocalizado. 
yuntura de un mundo cada vez más tecnológico. No conviene olvidar que este tipo de herramientas poseen un dinamismo innato que se genera por la actividad de los usuarios y que en ocasiones ha superado la funcionalidad para los que inicialmente fueron diseñadas.

Respecto a los procesos de participación online es importante remarcar que estas herramientas comunicativas representan una vía de conexión global con el mundo que resulta accesible y rápida. Internet, ha sido el epicentro de una revolución tecnológica que generalizado y estandarizado nuevas formas de comunicación. Formas que han permitido denunciar situaciones de regímenes dictatoriales o transgredir censuras, hasta el momento, infranqueables.

La participación del ser humano en Internet es un hecho que crece exponencialmente en todo el mundo, y es por ello, y atendiendo a nuestro compromiso profesional de afrontar los cambios sociales, por lo que el Trabajo Social como profesión debe replantear e incorporar parte de su práctica profesional, contemplando un contexto virtual cada vez más poblado. Un contexto en el que trascienden no solamente los problemas generados en Internet, sino otros problemas reales que precisan atención, asesoramiento e intervención profesional.

En el plano de la metodología de la intervención, uno de los principales inconvenientes que presentan este tipo de herramientas on-line afecta a la capacidad para establecer una buena relación empática con la persona demandante, sabiendo identificar los significados auditivos y visuales de la comunicación no verbal. Lo interesante de estas deficiencias es observar como el propio medio virtual dota de soluciones parciales a esta problemática, y actualmente el desarrollo de una infraestructura en la propia herramienta da lugar a videoconferencias con mayor calidad de imagen y sonido, que hacen subsanable en el futuro estos inconvenientes. Obviamente, la metodología de intervención virtual nunca llegará a remplazar el presencial; sin embargo, obviar las ventajas $\mathrm{y}$ potencialidades que presenta Internet como medio de comunicación, supone de algún modo anclarse en un modelo ortodoxo de intervención que cada vez más se aleja de la realidad de nuestros días.

\section{Bibliografía}

Arriazu, R. (2007). ¿Nuevos medios o nuevas formas de indagación?: Una propuesta metodológica para la investigación social on-line a través del foro de discusión, en Forum Qualitative Sozialforschung / Forum: Qualitative Social Research, (3), 1-15. Disponible en: http://nbn-resolving.de/urn:nbn:de:0114-fqs0703374

Barrueco et al. (1996). Las listas de correo como fuente de información en Biblioteconomía y documentación. Métodos de información, 3 (9), 22-29.

Bauman, Z. (2005). Modernidad líquida. Argentina: Fondo de Cultura Económica.

Bendit, R. (diciembre,1999). Participación Social y Política de los Jóvenes en Países de la Unión Europea. Trabajo presentado en Reunión de Trabajo de la Unión Europea «Juventud» CLACSO, Buenos Aires.

De Miguel, R. (2006). Fundamentos de la comunicación humana. Alicante: Club Universitario.

Krauskoff, D. (1998). Dimensiones críticas en la participación social de las juventudes: Participación y Desarrollo Social en la Adolescencia. San José: Fondo de Población de Naciones Unidas.

Ortoll, E. (2007). La alfabetización digital en los procesos de inclusión social. Barcelona: UOC.

O'Reilly, T. y Battelle, J. (2004). Opening Welcome: State of the Internet Industry. Cuadernos de Información y Comunicación. Madrid: Fundación Telefónica.

Peña, D. (2002). Cooperación y Voluntariado en red y en la Red. Documentación Social, 129, 186-203.

Prensky, M. (2001). Digital Natives, Digital Immigrants. On the Horizon, 9 , 1-6.

Toffler, A. y Toffler, H. (1996). La creación de una nueva civilización. La política de la tercera ola. México: Plaza \& Janes.

White, D y Le Cornu, A. (2011). Visitors and Residents: A new typology for online engagement. First Monday, 16 (9).

Disponible en: http://www.uic.edu/htbin/cgiwrap/bin/ojs/index.php/fm/article/viewArticle/3171/3049

Yus, F. (2001). Ciberpragmática. El uso del lenguaje en Internet. Barcelona: Ariel. 